\title{
Advanced Control Design of Four-Rotor Rotorcrafts
}

\author{
Tzu-Ching Chang ${ }^{2 *}$, and Yung-Yue Chen ${ }^{1}$, Bo-Hui Zhou ${ }^{3}$
}

\author{
${ }^{1}$ National Cheng Kung University, 1 University Road, Tainan City, 70101, Taiwan, R.O.C. \\ ${ }^{1}$ e-mail: yungyuchen@mail.ncku.edu.tw \\ ${ }^{2}$ National Cheng Kung University, 1 University Road, Tainan City, 70101, Taiwan, R.O.C. \\ 2e-mail: wysk2003@hotmail.com \\ ${ }^{3}$ No.16-2, Ln. 130, Sec. 1, Anzhong Rd., Annan Dist., Tainan City 709, Taiwan R.O.C. \\ 3e-mail: loki278478@gmail.com
}

\begin{abstract}
Four-Rotor rotorcrafts are widely applied in many purposes, such as military strategy, disaster relief, terrain investigation and environment exploration. Besides, FourRotor rotorcrafts have become the popular research because of the advantages of the better mobility, lower manufacture cost and smaller size than aircrafts. In this study, the system dynamic motion expressed by Lagrange method and the nonlinear control design of Four-Rotor rotorcrafts is based on feedback linearization method. The control inputs: total thrust and torques can be derived elegantly by taking the position errors and attitude angle errors of Four-Rotor rotorcrafts into consideration, and the errors can be proven to converge to zero asymptotically. By the simulation results, the effectiveness of this control law is demonstrated that all the tracking errors converge to near zero. Finally, FourRotor rotorcrafts will track the waypoints precisely by various numerical simulations and practical verification under different flight initial conditions.
\end{abstract}

Keywords: Four-Rotor rotorcrafts, Feedback linearization, Nonlinear control.

\section{Introduction}

Compared with the usual Unmanned aerial vehicles (UAVs), the most important advantage that a Four-Rotor rotorcraft is its mobility, i.e., it can fly toward any direction freely. The difference between conventional helicopters and Four-Rotor rotorcrafts is their flight control methods. A helicopter is controlled by changing the angle of attack of the rotor whereas a Four-Rotor rotorcraft is controlled by tuning the speed of four rotors. Four-Rotor rotorcrafts have four rotors. They can generate better thrust than conventional helicopters and make stable hovering possible.

In order to compensate the effect of the reactive torques, the four rotors are divided into two pairs of $(1,3)$ and $(2,4)$ turning in the opposite direction, as depicted in Fig. 1. Four-Rotor rotorcraft is a typical under-actuated, non-linear coupled system, and it has six degrees-offreedom (DOF) that involves three translations and three rotations: vertical motion created by collectively increasing and decreasing the speed of all four rotors; pitch or roll motion is achieved by the differential speed of the $(1,3)$ set or the $(2,4)$ set of rotors, coupled with lateral motion; yaw motion is implemented by the different reactive torques between the $(1,3)$ and the $(2,4)$ rotors. So the number of individual manipulating variables cannot instantaneously set the accelerations in all directions of the configuration space.

In the past decade, McKerrow ${ }^{(1)}$ studied the dynamical model of the fourrotor VTOL vehicles in the literature. Hamel et al. ${ }^{(2)}$ made a slight modification of the gyroscopic torques expression on the dynamical model in ${ }^{(1)}$ and designed a backstepping-based trajectory tracking controller. Tayebi and McGilvray ${ }^{(3)}$ propose a new quaternion-based feedback control scheme for exponential attitude stabilization of vertical takeoff and landing of Four-Rotor rotorcrafts, and the proposed controller is based upon the compensation of the Coriolis and gyroscopic torques and the use of a $\mathrm{PD}^{2}$ feedback structure. Zuo, Z. ${ }^{(4)}$, based on the relationship between the attitude and linear acceleration of a small Four-Rotor rotorcraft to deign a new command-filtered backstepping technique to stabilise the attitude and further developed a linear tracking differentiator to eliminate the classical inner/outer-loop structure. Pedro Castillo ${ }^{(5)}$ proposed a controller based on Lyapunov analysis with a nested saturation algorithm, and the controller is able to perform autonomously the tasks of taking off, hovering, and landing. Bouabdallah et al. ${ }^{(6)}$ applied the classical PID and LQ algorithm, respectively, to the quadrotor attitude stabilisation.

In this study, the three translations and the three rotations of the dynamic model are discussed separately. Firstly, based on the position errors, the desired attitude angle and total thrust can be derived. Then, using the attitude angle errors, we can design the control torques by feedback linearization method. Finally, the errors can be proven to converge to zero asymptotically.

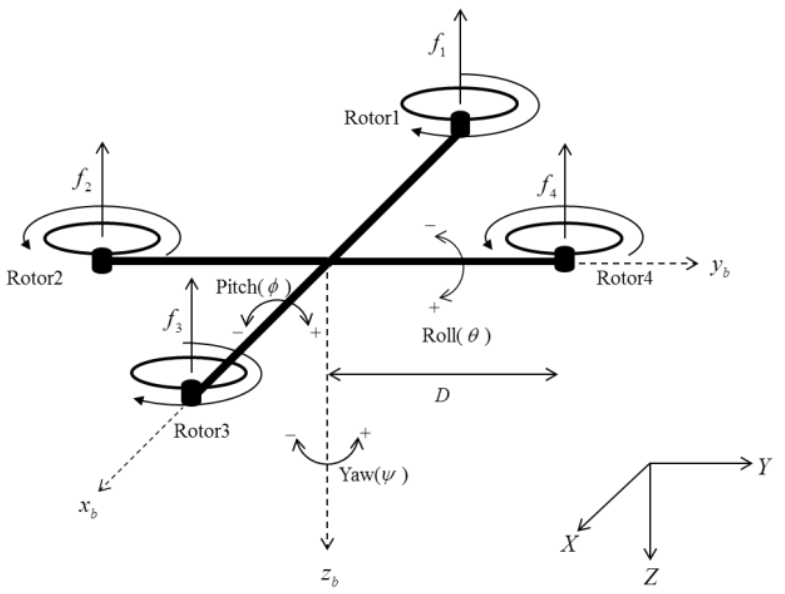

Fig. 1 Schema of the four-rotor rotorcraft 


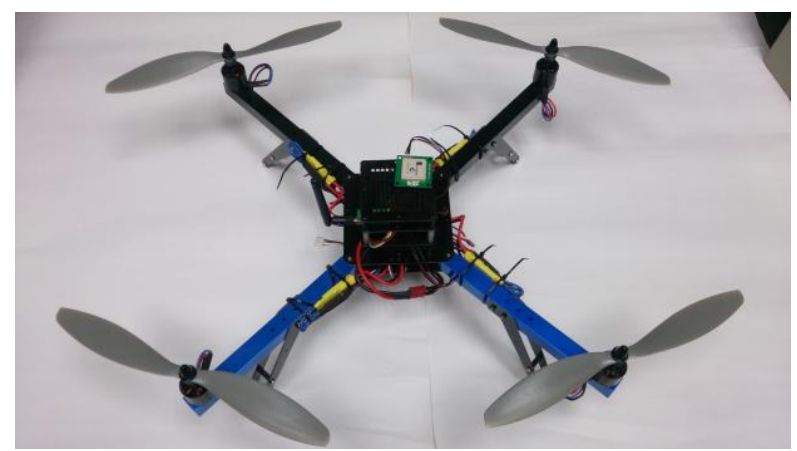

Fig. 2 Four-Rotor rotorcraft

\section{Dynamic Equations of Four-Rotor Rotorcrafts Structure}

The practical implementation of this proposed investigation is with the four-Rotor rotorcraft given as Fig. 2.

Generally, there are two methods can be utilized to derive the model of Four-Rotor rotorcrafts. The first one is Newton method and the other is Lagrange method. In this investigation, Lagrange method is adopted to derive the system dynamic model. A generalized coordinates can be expressed for Four-Rotor rotorcrafts:

$q=(X, Y, Z, \psi, \theta, \phi) \in \mathfrak{R}^{6}$

where $p=(X, Y, Z) \in \mathfrak{R}^{3}$ represent the position vector of the Four-Rotor rotorcraft in Earth-frame and $\Theta=(\psi, \theta, \phi) \in \mathfrak{R}^{3}$ are the Euler angle vector ( $\psi$ is the yaw angle, $\theta$ is the roll angle and $\phi$ is the pitch angle) which represent the orientation of the controlled FourRotor rotorcraft.

By Lagrange method, the translational kinetic energy, rotational kinetic energy and potential energy for the Four-Rotor rotorcraft can be described as follows:

$T_{\text {trans }}=\frac{m}{2} \dot{p}^{T} \dot{p}$

$T_{r o t}=\frac{1}{2} \omega^{T} I \omega$

$U=m g Z$

where $\omega$ denote the angular rates, $I$ is the inertia matrix, $m$ is the mass, $Z$ is height of Four-Rotor rotorcrafts and $g$ denotes gravity.

Relationship between the systems angular rates and Euler rates can be represented as:

$\omega=W \dot{\Theta}$

Where

$W=\left[\begin{array}{ccc}-\sin \theta & 0 & 1 \\ \cos \theta \sin \phi & \cos \phi & 0 \\ \cos \theta \cos \phi & -\sin \phi & 0\end{array}\right]$.
By substituting Eq. (4) to Eq. (2), we have:

$T_{r o t}=\frac{1}{2}\left(\dot{\psi}^{2}+\dot{\theta}^{2}+\dot{\phi}^{2}\right)-\dot{\psi} \dot{\phi} \sin \theta$

The Lagrangian is defined as the following:

$L(q, \dot{q})=T_{\text {trans }}+T_{\text {rot }}-U$

The full dynamic equations of the Four-Rotor rotorcraft can be obtained by combining Euler-Lagrange equations and the generalized force balance concept.

$\frac{d}{d t} \frac{\partial L}{\partial \dot{q}}-\frac{\partial L}{\partial q}=\left[\begin{array}{c}T_{p} \\ \tau\end{array}\right]$

where $T_{p}$ is translational force acts on the Four-Rotor rotorcraft, and can be described as:

$T_{p}=R \hat{T}$

where $R$ is the transformation matrix from body-frame to Earth-frame as below:

$R=\left[\begin{array}{ccc}c_{\theta} c_{\psi} & c_{\theta} s_{\psi} & -\mathrm{s}_{\theta} \\ s_{\theta} c_{\psi} s_{\phi}-s_{\psi} c_{\phi} & s_{\theta} s_{\psi} s_{\phi}+c_{\psi} c_{\phi} & c_{\theta} s_{\phi} \\ s_{\theta} c_{\psi} c_{\phi}+s_{\psi} s_{\phi} & s_{\theta} s_{\psi} c_{\phi}-c_{\psi} s_{\phi} & c_{\theta} c_{\phi}\end{array}\right]$

where $s_{(\cdot)}=\sin (\cdot)$ and $c_{(\cdot)}=\cos (\cdot)$

and $\hat{T}$ denotes the thrust vector represented as $\hat{T}=\left[\begin{array}{l}0 \\ 0 \\ u\end{array}\right]=\left[\begin{array}{c}0 \\ 0 \\ \sum_{i=1}^{4} f_{i}\end{array}\right]=\left[\begin{array}{c}0 \\ 0 \\ \sum_{i=1}^{4} b \omega_{i}\end{array}\right]$

where $b$ is a positive constant represents thrust coefficient and $\omega_{i}$, for $i=1, . ., 4$ are the rotation speed of rotor " $i$ ".

The generalized torque $\tau$ is described as follows:

$\tau=\left[\begin{array}{c}\tau_{\psi} \\ \tau_{\theta} \\ \tau_{\phi}\end{array}\right]=\left[\begin{array}{c}\sum_{i=1}^{4} \tau_{M i} \\ D\left(f_{2}-f_{4}\right) \\ D\left(f_{3}-f_{1}\right)\end{array}\right]$

where $\tau_{M i}$ is the couple moment of motor $M_{i}$ and $D$ is the distance between the center of each mass and rotor.

The movement patterns of $\tau_{\psi}, \tau_{\theta}$ and $\tau_{\phi}$ are shown as Fig. 3, Fig. 4 and Fig. 5, respectively. 


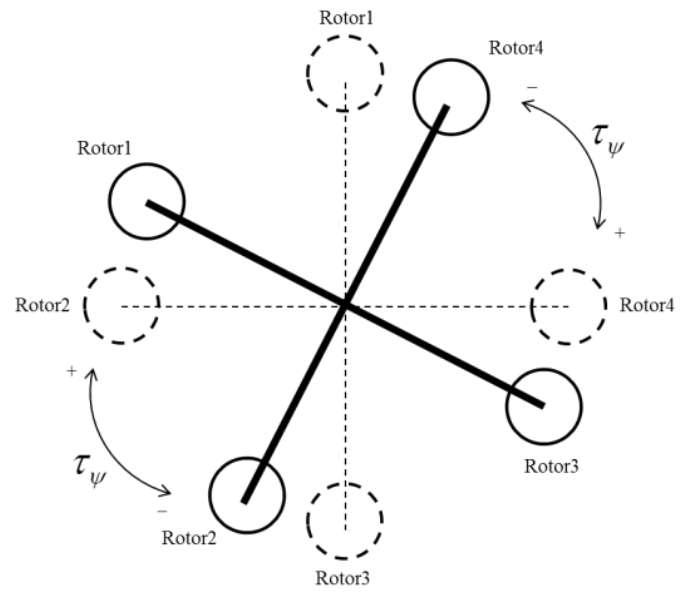

Fig. 3 Schematic diagram of $\tau_{\psi}$

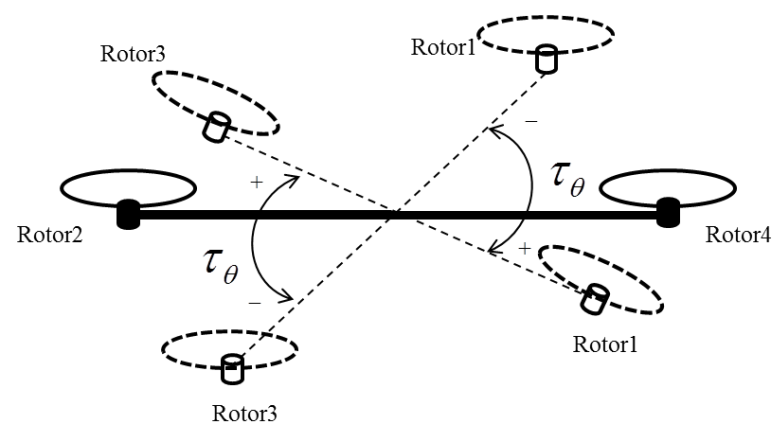

Fig. 4 Schematic diagram of $\tau_{\theta}$

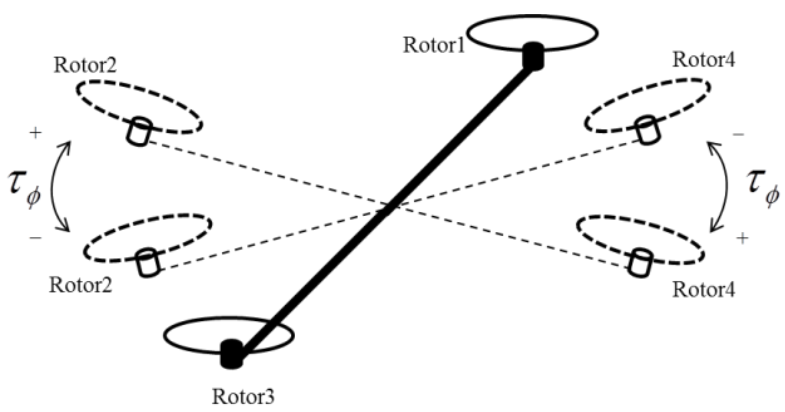

Fig. 5 Schematic diagram of $\tau_{\phi}$

By substituting (6) to (7) and combining (10) and (11), the dynamic equations of Four-Rotor rotorcraft can be expressed as:

$$
\begin{aligned}
\ddot{X} & =-u \frac{1}{m} \sin \theta \\
\ddot{Y} & =\frac{1}{m} u(\cos \theta \sin \phi) \\
\ddot{Z} & =\frac{1}{m} u(\cos \theta \cos \phi)-g \\
\ddot{\psi} & =\sum_{i=1}^{4} \tau_{M i}+\ddot{\phi} \sin \theta+\dot{\phi} \dot{\theta} \cos \theta \\
\ddot{\theta} & =D\left(f_{2}-f_{4}\right)-\dot{\psi} \dot{\phi} \cos \theta \\
\ddot{\phi} & =D\left(f_{3}-f_{1}\right)+\ddot{\psi} \sin \theta+\dot{\psi} \dot{\theta} \cos \theta
\end{aligned}
$$

\section{Control Law Design}

Define tracking errors:

$e_{1}=p-p_{d}$

where $p_{d}=\left[\begin{array}{c}X_{d} \\ Y_{d} \\ Z_{d}\end{array}\right]$ is the desired waypoint.

For achieving this target, feedback linearization method is adopted for the tracking error convergence purpose, and a second order differential error equation can be derived as:

$\ddot{e}_{1}+K_{d} \dot{e}_{1}+K_{p} e_{1}=0$

Where $K_{d 1}$ and $K_{p 1}$ are control parameter matrices as below with adjustable coefficients $\lambda_{i}$, for $i=1, \cdots, 6$.

$K_{d 1}=\left[\begin{array}{ccc}\lambda_{1} & 0 & 0 \\ 0 & \lambda_{2} & 0 \\ 0 & 0 & \lambda_{3}\end{array}\right]$ and $K_{p 1}=\left[\begin{array}{ccc}\lambda_{4} & 0 & 0 \\ 0 & \lambda_{5} & 0 \\ 0 & 0 & \lambda_{6}\end{array}\right]$

Because Eq. (14) satisfies Routh Hurwitz condition, it guarantees $\lim _{t \rightarrow \infty} e_{1}(t)=0$ exponentially.

Define a virtual control command $\bar{U}$ as:

$$
\bar{U}=\left[\begin{array}{c}
\bar{U}_{1} \\
\bar{U}_{2} \\
\bar{U}_{3}
\end{array}\right]=\ddot{p}=\left[\begin{array}{c}
\ddot{X} \\
\ddot{Y} \\
\ddot{Z}
\end{array}\right]=\left[\begin{array}{c}
-\frac{1}{m} \sin \theta \\
\frac{1}{m}(\cos \theta \sin \phi) \\
\frac{1}{m}(\cos \theta \cos \phi)-g
\end{array}\right]
$$

From Eq. (13) and Eq. (14), we have

$\ddot{p}+K_{d} \dot{p}+K_{p}\left(p-p_{d}\right)=0$

Based on (15) and (16), the virtual control command $\bar{U}$ is chosen as the following:

$\bar{U}=-K_{d} \dot{p}-K_{p}\left(p-p_{d}\right)$

Eq. (15) can be further written as:

$\ddot{p}=\left[\begin{array}{c}\bar{U}_{1} \\ \bar{U}_{2} \\ \bar{U}_{3}\end{array}\right]=-g z_{e}+\frac{1}{m} u R z_{e}$

where $z_{e}$ is the unit vector of the $Z$ axis in Earth coordinate.

Rearranging Eq. (18), we have:

$R^{T}\left(\ddot{p}+g z_{e}\right)=\frac{u}{m} z_{e}$

i.e., 
$\left[\begin{array}{ccc}c_{\theta} c_{\psi} & s_{\theta} c_{\psi} s_{\phi}-s_{\psi} c_{\phi} & s_{\theta} c_{\psi} c_{\phi}+s_{\psi} s_{\phi} \\ c_{\theta} s_{\psi} & s_{\theta} s_{\psi} s_{\phi}+c_{\psi} c_{\phi} & s_{\theta} s_{\psi} c_{\phi}-c_{\psi} s_{\phi} \\ -\mathrm{s}_{\theta} & c_{\theta} s_{\phi} & c_{\theta} c_{\phi}\end{array}\right]\left[\begin{array}{c}\bar{U}_{1} \\ \bar{U}_{2} \\ \bar{U}_{3}+g\end{array}\right]=\left[\begin{array}{c}0 \\ 0 \\ \frac{u}{m}\end{array}\right]$

where $s_{(\cdot)}=\sin (\cdot)$ and $c_{(\cdot)}=\cos (\cdot)$.

The relationship between $\bar{U}$ and $u$ can be calculated easily, and it can be described as the following:

$u=m\left[-\bar{U}_{1}\left(\mathrm{~s}_{\theta}\right)+\bar{U}_{2}\left(c_{\theta} s_{\phi}\right)+\left(\bar{U}_{3}+g\right)\left(c_{\theta} c_{\phi}\right)\right]$

A sufficient condition for obtaining the desired pitch angle $\phi$ by dividing $\bar{U}_{2}$ with $\bar{U}_{3}$ can be given as:

$\phi_{d}=\tan ^{-1}\left(\frac{\bar{U}_{2}}{\bar{U}_{3}+g}\right)$

Dividing $\bar{U}_{1}$ with $\bar{U}_{2}$, we have:

$\frac{\bar{U}_{1}}{\bar{U}_{2}}=\frac{-\tan \theta}{\cos \phi_{d}}$

Substituting Eq. (21) into Eq. (2), we obtain the second sufficient condition for obtaining the desired row angle as the following:

$\theta_{d}=\tan ^{-1}\left(\frac{\bar{U}_{1}}{\sqrt{\left(\bar{U}_{3}+g\right)^{2}+\left(\bar{U}_{2}\right)^{2}}}\right)$

Based on the above derivations, the controlled attitude angles are given as:

$\Theta_{d}=\left[\begin{array}{c}\psi_{d} \\ \theta_{d} \\ \phi_{d}\end{array}\right]=\left[\begin{array}{c}\psi_{d} \\ \tan ^{-1}\left(\frac{\bar{U}_{1}}{\left(\bar{U}_{3}+g\right)^{2}+\left(\bar{U}_{2}\right)^{2}}\right) \\ \tan ^{-1}\left(\frac{\bar{U}_{2}}{\bar{U}_{3}+g}\right)\end{array}\right]$

where $\psi_{d}$ is desired LOS angle.

Define the attitude errors:

$e_{2}=\Theta-\Theta_{d}$

where $\Theta=\left[\begin{array}{c}\psi \\ \theta \\ \phi\end{array}\right]$ and $\Theta_{d}=\left[\begin{array}{c}\psi_{d} \\ \theta_{d} \\ \phi_{d}\end{array}\right]$.

By feedback linearization method, we have:

$\ddot{e}_{2}+\bar{K}_{d} \dot{e}_{2}+\bar{K}_{p} e_{2}=0$

By selecting:
$\ddot{\Theta}=\left[\begin{array}{c}\ddot{\psi} \\ \ddot{\theta} \\ \ddot{\phi}\end{array}\right]=c+\tau$

where $c=\left[\begin{array}{c}\ddot{\phi} \sin \theta+\dot{\phi} \dot{\theta} \cos \theta \\ -\dot{\psi} \dot{\phi} \cos \theta \\ \ddot{\psi} \sin \theta+\dot{\psi} \dot{\theta} \cos \theta\end{array}\right]$, and $\tau=\left[\begin{array}{c}\tau_{\psi} \\ \tau_{\theta} \\ \tau_{\phi}\end{array}\right]=\left[\begin{array}{c}\sum_{i=1}^{4} \tau_{M i} \\ D\left(f_{2}-f_{4}\right) \\ D\left(f_{3}-f_{1}\right)\end{array}\right]$.

and for tracking $\Theta_{d}$, the controller can be chosen as:

$$
\begin{aligned}
\tau & =\left[\begin{array}{c}
\ddot{\psi}_{d} \\
\ddot{\theta}_{d} \\
\ddot{\phi}_{d}
\end{array}\right]-\left[\begin{array}{c}
\ddot{\phi} \sin \theta+\dot{\phi} \dot{\theta} \cos \theta \\
-\dot{\psi} \dot{\phi} \cos \theta \\
\ddot{\psi} \sin \theta+\dot{\psi} \dot{\theta} \cos \theta
\end{array}\right] \\
& -K_{d 2}\left[\begin{array}{c}
\dot{\psi}-\dot{\psi}_{d} \\
\dot{\theta}-\dot{\theta}_{d} \\
\dot{\phi}-\dot{\phi}_{d}
\end{array}\right]-K_{p 2}\left[\begin{array}{c}
\psi-\psi_{d} \\
\theta-\theta_{d} \\
\phi-\phi_{d}
\end{array}\right]
\end{aligned}
$$

where $K_{d 2}$ and $K_{p 2}$ are control parameter matrices with adjustable coefficients $\sigma_{i}$, for $i=1, \cdots, 6$.

$$
K_{d 2}=\left[\begin{array}{ccc}
\sigma_{1} & 0 & 0 \\
0 & \sigma_{2} & 0 \\
0 & 0 & \sigma_{3}
\end{array}\right] \text {, and } K_{p 2}=\left[\begin{array}{ccc}
\sigma_{4} & 0 & 0 \\
0 & \sigma_{5} & 0 \\
0 & 0 & \sigma_{6}
\end{array}\right]
$$

Because Eq. (28) satisfies Routh Hurwitz condition, it guarantees $\lim _{t \rightarrow \infty} e_{2}(t)=0$ exponentially.

According to the above mentions, the Block diagram of the Four-Rotor rotorcraft design process is shown as Fig. 5.

Finally, the Four-Rotor rotorcraft can be controlled precisely to approach the desired waypoints.

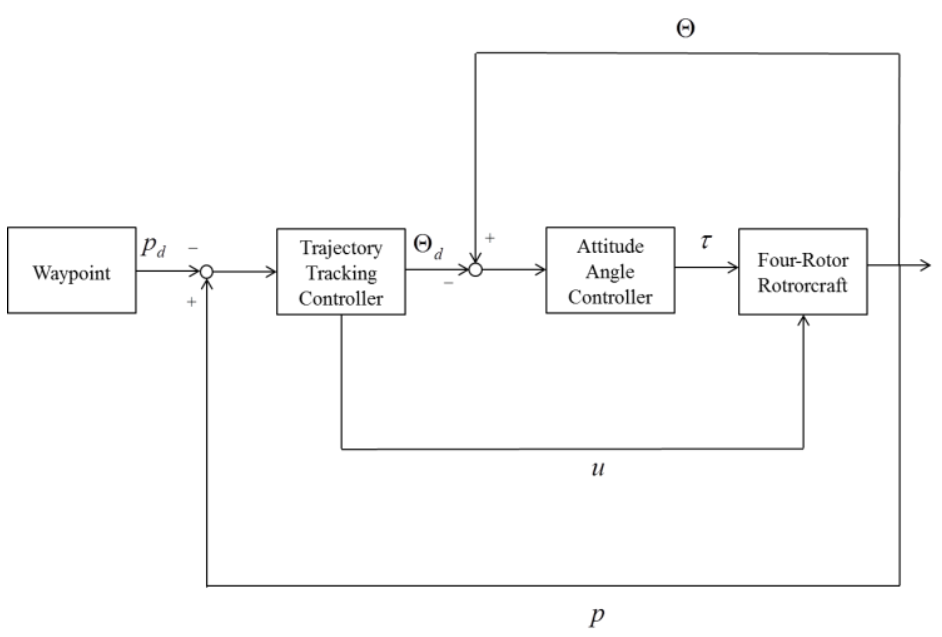

Fig. 5 Block diagram of the Four-Rotor rotorcrafts design process 


\section{Simulation Results}

The design objective of the control of the Four-Rotor rotorcraft is to control the attitude angles of Four-Rotor rotorcraft to precisely approach the desired attitude angles and positions of the arranged waypoints. For verifying the performances of this proposed control law, the simulation results were implemented by Matlab software.

A simple and monotonic path for testing the tracking performance of proposed control law and predefined waypoints are shown as Table 1. In Figs. 6, the trajectory history is exhibited on the 3-D space. By analyzing these trajectories, we can see that the Four-Rotor rotorcraft fly to the defined waypoint successfully. The tracking errors of positions in $\mathrm{X}, \mathrm{Y}$ and $\mathrm{Z}$ directions are shown as Figs. 79 and the tracking errors of attitude angles are shown as Figs. 10-12. From Figs. 7-12, all the tracking errors converge to near zero.

Table. 1 Desired waypoints

\begin{tabular}{|c|c|c|c|}
\hline No. & Waypoint $(X, Y, Z)$ & No. & Waypoint $(X, Y, Z)$ \\
\hline 0 & $(0,0,0)$ & 3 & $(50,50,50)$ \\
\hline 1 & $(0,0,50)$ & 4 & $(0,50,50)$ \\
\hline 2 & $(50,0,50)$ & 5 & $(0,0,0)$ \\
\hline
\end{tabular}

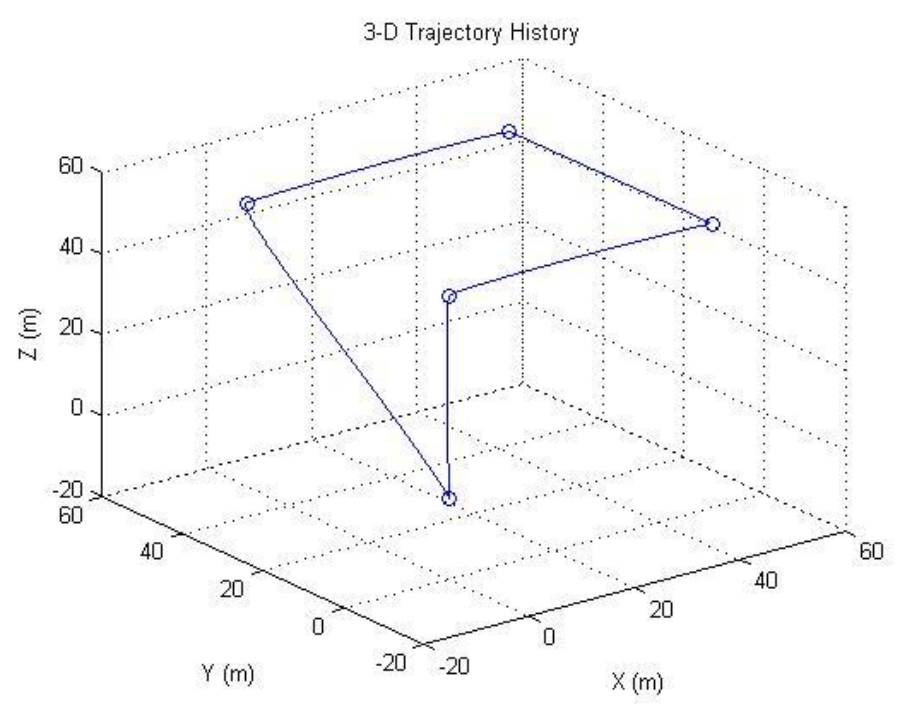

Fig. 6 The 3-D flight trajectory of the Four-Rotor rotorcraft

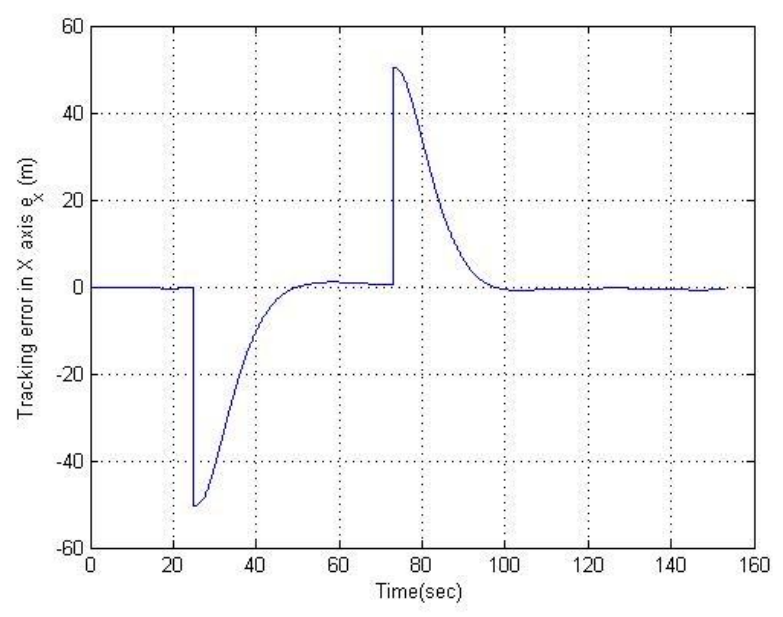

Fig. 7 The tracking errors in $X$ axis $e_{X}(\mathrm{~m})$

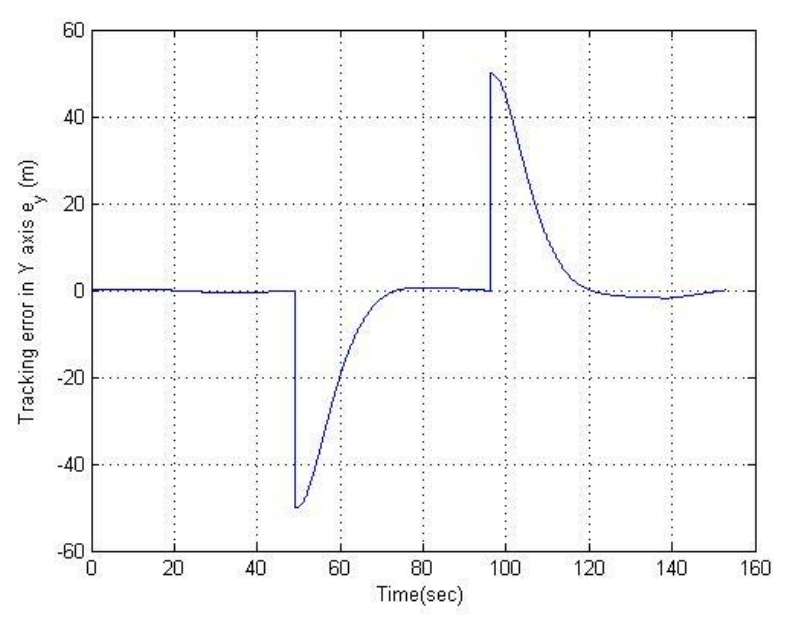

Fig. 8 The tracking errors in $Y$ axis $e_{Y}(\mathrm{~m})$

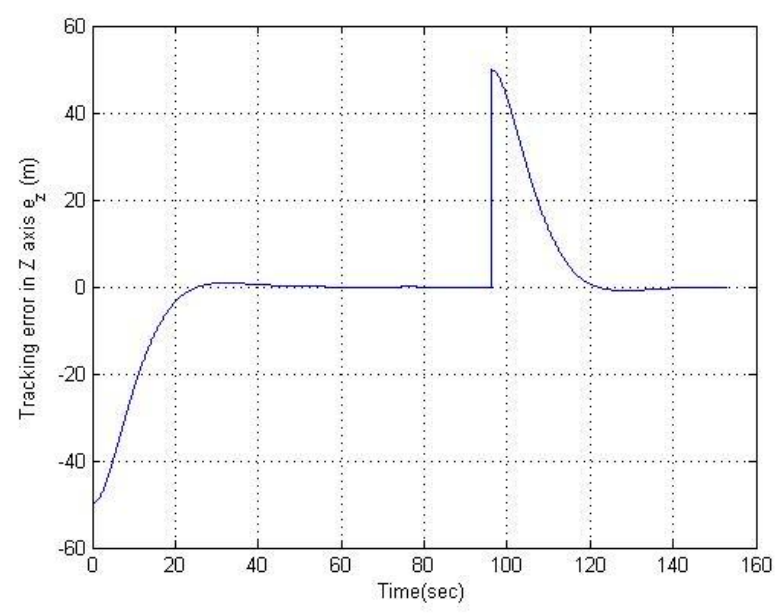

Fig. 9 The tracking errors in $Z$ axis $e_{Z}(\mathrm{~m})$ 


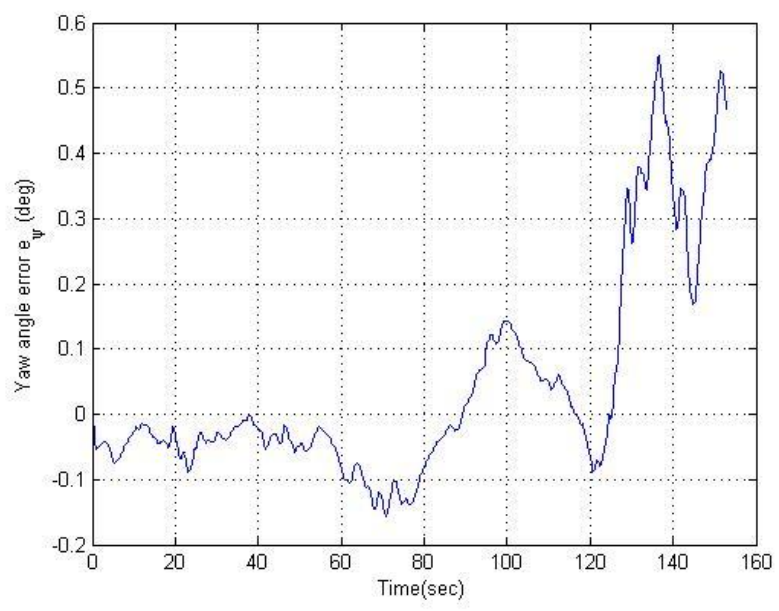

Fig. 10 The tracking errors of yaw angle

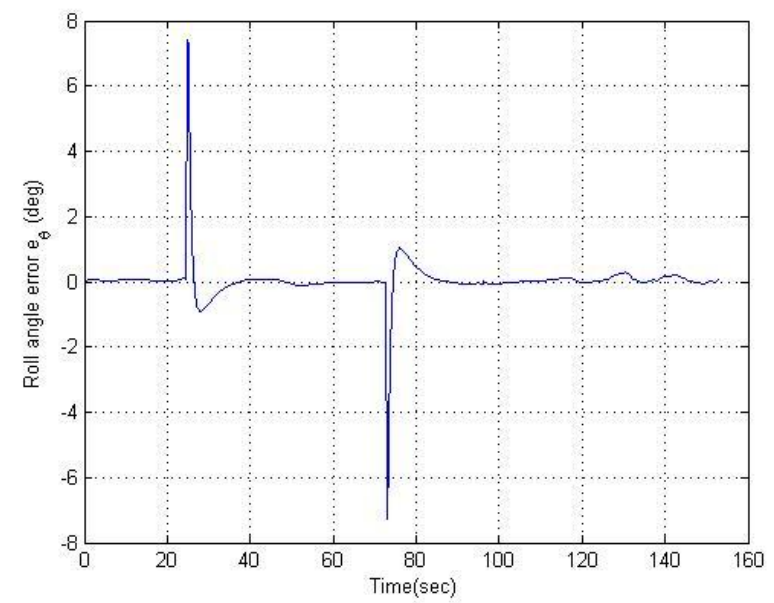

Fig. 11 The tracking errors of roll angle

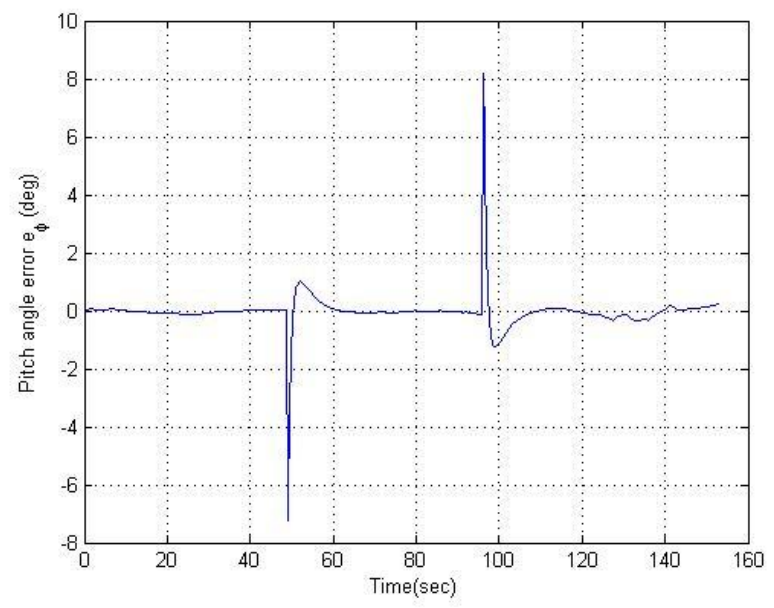

Fig. 12 The tracking errors of pitch angle

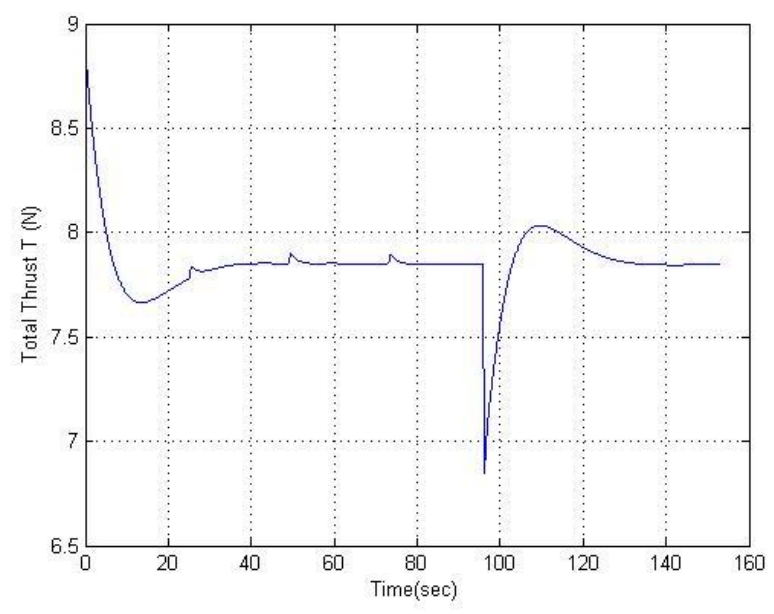

Fig. 13 The history of total thrust

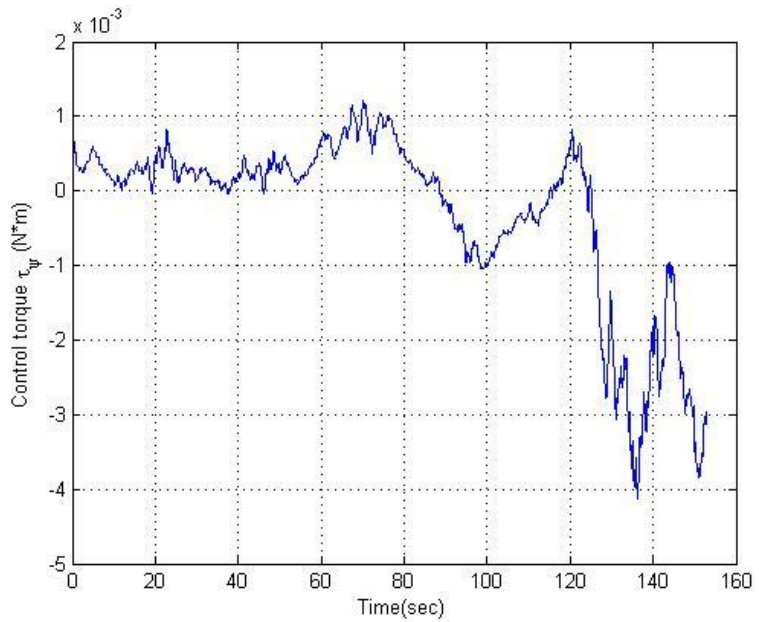

Fig. 14 The control torque in rotation of yaw

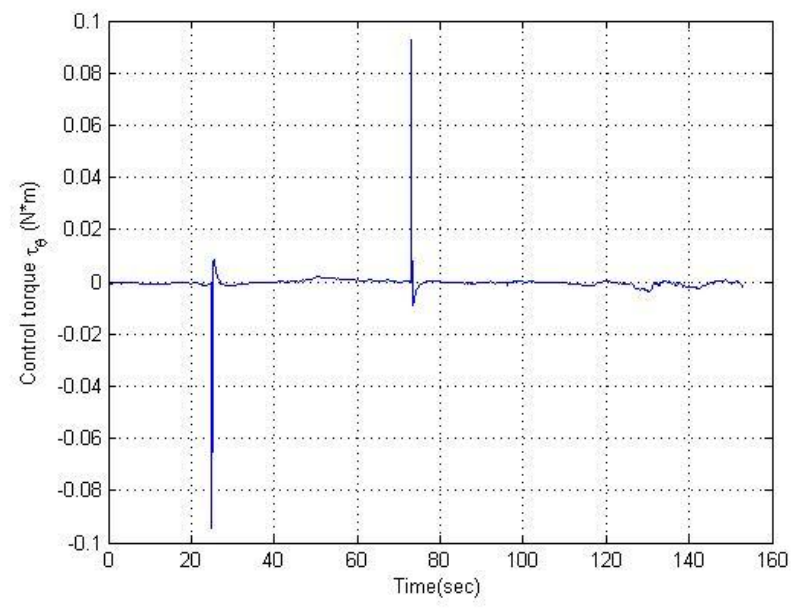

Fig. 15 The control torque in rotation of roll 


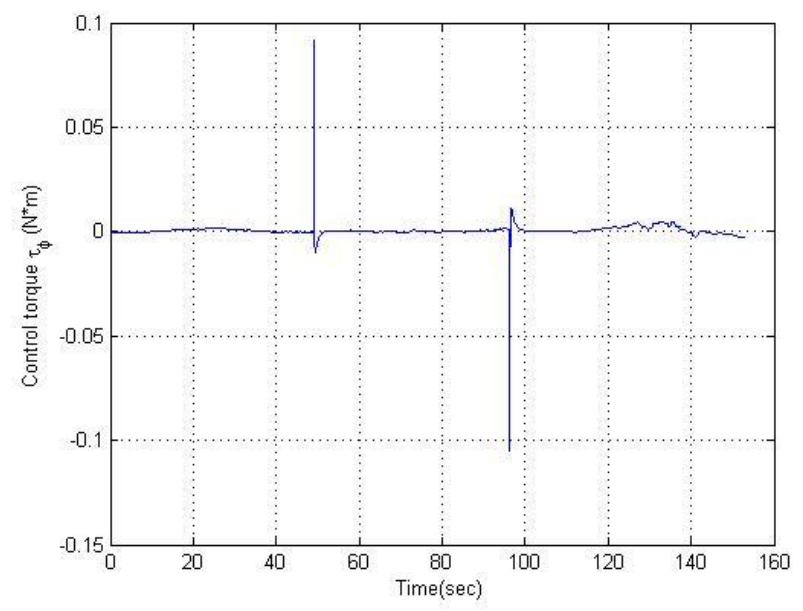

Fig. 16 The control torque in rotation of pitch

\section{Conclusions}

In this investigation, the dynamic model of Four-Rotor rotorcraft is derived by Lagrange method, and then the desired attitude angles, thrust and control torques for precisely guiding the Four-Rotor rotorcraft to the predefined waypoints can be elegantly derived as the proposed method which is based on the concept of feedback linearization method.

From the above simulation results, the Four-Rotor rotorcraft can be precisely guided to the predefined waypoints with a profile as a point to point straight trajectory, and all the tracking errors converge to near zero by this proposed method.

\section{References}

(1) P. McKerrow, "Modelling the Dragon flyer four-rotor helicopter", Proceedings of the IEEE International Conference on Robotics and Automation, USA, 2004.

(2) T. Hamel, R. Mahony, R. Lozano and J. Ostrowski, "Dynamic Modelling and Configuration Stabilization for an X4-Flyer", Proc. IFAC World Congress, Barcelona, Spain, 2002.

(3) A. Tayebi and S. McGilvray, "Attitude Stabilization of a VTOL Quadrotor Aircraft", IEEE Trans. Control Syst. Technol., Vol. 14, No. 3, pp. 562-572, 2006.

(4) Z. Zuo, "Trajectory tracking control design with command-filtered compensation for a quadrotor", IET Control Theory and Applications, Vol. 4, No. 11, pp. 2343-2355, 2010.

(5) P. Castillo, A. Dzul, and R. Lozano, "Real-time stabilization and tracking of a four rotor mini rotorcraft", IEEE Trans. Control Syst. Technol., Vol. 12, No. 4, pp. 510-516, 2004.

(6) S. Bouabdallah, A. Noth, and R. Siegwan, "PID vs LQ control techniques applied to an indoor micro quadrotor", Proc. 2004 IEEE/RSJ Int. Conf. Intelligent Robots and Systems, pp. 2451-2456, Sandal, Japan, 2004.
(7) A. Das, K. Subbarao, and F. Lewis, "Dynamic inversion with zero-dynamics stabilization for quadrotor control", IET Control Theory and Applications, Vol. 3, No. 3, pp. 303-314, 2009.

(8) J. T.-Y. Wen and K. Kreutz-Delgado, "The attitude control problem", IEEE Trans. Autom. Control, Vol. 36, No. 10, pp. 1148-1162, 1991.

(9) I. Kanellakopoulos, P.V. Kokotovic, A.S. Morse, "Systematic design of adaptive controllers for feedback linearizable systems", IEEE Trans. Autom. Control, Vol. 36, No. 11, pp. 1241-1253, 1991.

(10) F. Lizarralde and J. T. Wen, "Attitude Control Without Angular Velocity Measurement: A Passivity Approach", IEEE Trans. Autom. Control, Vol. 41, No. 3, pp. 468-472, 1996. 